\title{
The proposed algorithm for emergency endoscopy during the coronavirus disease 2019 outbreak
}

\author{
Sung Bum Kim and Kook Hyun Kim
}

Division of Gastroenterology and Hepatology, Department of Internal medicine, Yeungnam University College of Medicine, Daegu, Korea

\author{
Received: May 14, 2020 \\ Revised : June 12, 2020 \\ Accepted: June 13, 2020 \\ Correspondence to \\ Kook Hyun Kim, M.D. \\ Department of Internal \\ Medicine, Yeungnam University \\ College of Medicine, 170 \\ Hyeonchung-ro, Nam-gu, Daegu \\ 42415 , Korea \\ Tel: $+82-53-620-3576$ \\ Fax: +82-53-654-8386 \\ E-mail: kimkh@yu.ac.kr \\ https://orcid.org/0000-0001- \\ 7786-7882
}

Following the coronavirus disease 2019 outbreak in China, more than 10,765 patients tested positive for severe acute respiratory syndrome coronavirus 2 from February 18, 2020 to April 30, 2020 in South Korea. Performing emergency endoscopy is extremely challenging from the clinicians' viewpoint during the viral outbreak. There are no available guidelines for emergency endoscopy in tertiary hospitals during this pandemic. We set up an algorithm as a guide for emergency endoscopy in patients presenting to the emergency room with bleeding, foreign body, or impending cholangitis. From February 18, 2020 to April 30, 2020 of outbreak, 130 patients underwent emergency endoscopy in our center. Owing to the simple and streamlined algorithm for performing emergency endoscopy, no endoscopy-related infection to other patients or medical workers was reported in our center.

Keywords: Coronavirus; COVID-19; Endoscopy
Following the coronavirus disease 2019 (COVID-19) outbreak in China, a sudden regional COVID-19 outbreak occurred in Daegu, South Korea [1]. The surge of confirmed COVID-19 patients may influence the treatment modality for patients who visit the emergency room (ER) presenting with abdominal pain, gastrointestinal bleeding, acute cholangitis, or foreign body impaction in the gastrointestinal tract. The highly virulent severe acute respiratory syndrome coronavirus 2 (SARS-CoV-2) is well known to be transmitted via the respiratory tract by aerosol droplets. However, fecal to oral transmission has been postulated; therefore, endoscopy may pose a risk for transmission [2]. Protection of medical workers and prevention of viral spread to other patients are critical issues that need to be monitored while performing endoscopic procedures in the endoscopy room, ER, and intensive care unit of tertiary hospitals $[3,4]$. More than
10,765 patients tested positive for SARS-CoV-2 from February 18, 2020 to April 30, 2020 in South Korea with most cases originating from a specific religious group within Daegu. During this period, most routine endoscopies were canceled or postponed in order to prevent nosocomial spread, based on the infection control policy of Yeungnam University Hospital [3]. There is no available guideline for endoscopy in the ER of a tertiary hospital during the COVID-19 pandemic. Therefore, we set up an algorithm as a guide for conducting endoscopy, including gastrofibroscopy, sigmoidoscopy, colonoscopy, and endoscopic retrograde cholangiopancreatography (ERCP) (Fig. 1). Those who visited the ER immediately underwent screening for fever and respiratory symptoms (cough, sputum, or sore throat) and intensive history-taking on whether the patient is a Shinchungi religious churchgoer, recent visit to China, or a close contact 


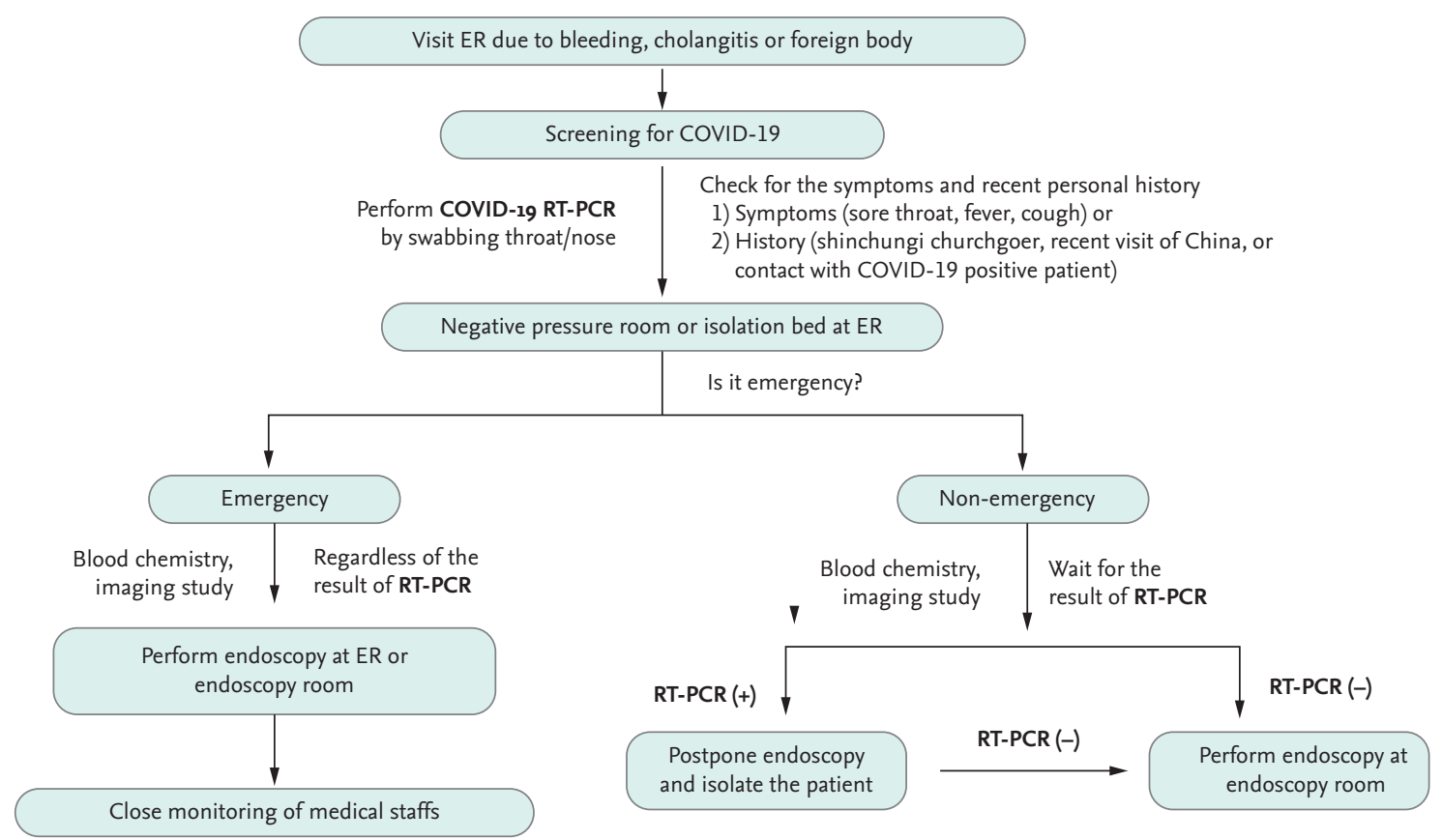

Figure 1. Algorithm for management of patients requiring emergency endoscopy during the coronavirus disease 2019 (COVID-19) outbreak. This algorithm describes how we manage a patient with gastrointestinal emergency in the emergency room (ER). Patients undergo intensive history-taking and assessment for the presence of flu-like symptoms. After swabbing the throat/nose for reverse-transcription polymerase chain reaction (RT-PCR), patients are quarantined at the isolation room in the ER until the RT-PCR test results are out. In cases of emergency, endoscopy can be performed immediately, regardless of RT-PCR results. If not, however, endoscopy should be delayed until RT-PCR result is confirmed; then, an expected endoscopic management is planned based on the PCR results.

episode with a SARS-CoV-2-positive patient. Those who had fever, respiratory symptoms, or a pertinent history underwent reverse-transcription polymerase chain reaction (RT-PCR) testing for SARS-CoV-2 using throat and nose swabs and/or sputum, which takes approximately 4 to 6 hours to obtain results on weekdays and 12 hours on weekends. While waiting for RT-PCR results, patients were transferred to an isolated or negative pressure room. Patients suspected of having COVID-19 infection and necessitated to undergo emergency endoscopy due to hematemesis, foreign body exposure, or ongoing cholangitis subsequently underwent emergency endoscopy based on clinicians' discretion. All endoscopies for patients suspected of having COVID-19 infection and without confirmed negative RT-PCR results were performed under level D protection. From February 18, 2020 to April 30, 2020, a total of 130 patients underwent endoscopy through the ER in our center:
ERCP was performed in 54 cases, gastrofibroscopy in 67 cases, colonoscopy in three cases, and sigmoidoscopy in nine cases. Endoscopy was performed under level D protection in two patients. Among these 130 patients, 40 (30.8\%) had fever and respiratory symptoms or possibility of a close contact episode with a SARS-CoV-2-positive patient. Only one patient who was initially afebrile was diagnosed with COVID-19 14 days after ERCP. Performing endoscopy is a major challenge during the viral outbreak [3-5]. All patients were required to wear masks before and after gastrofibroscopy or ERCP and were only unmasked while undergoing gastrofibroscopy or ERCP. All medical personnel in endoscopy units wear personal protective equipment including N95 mask, hair cap, two layers of surgical gloves, goggles, face shield, waterproof gown, and shoe covers when performing emergent endoscopy in patients with fever, respiratory symptoms or those who had possible close contact episodes with 


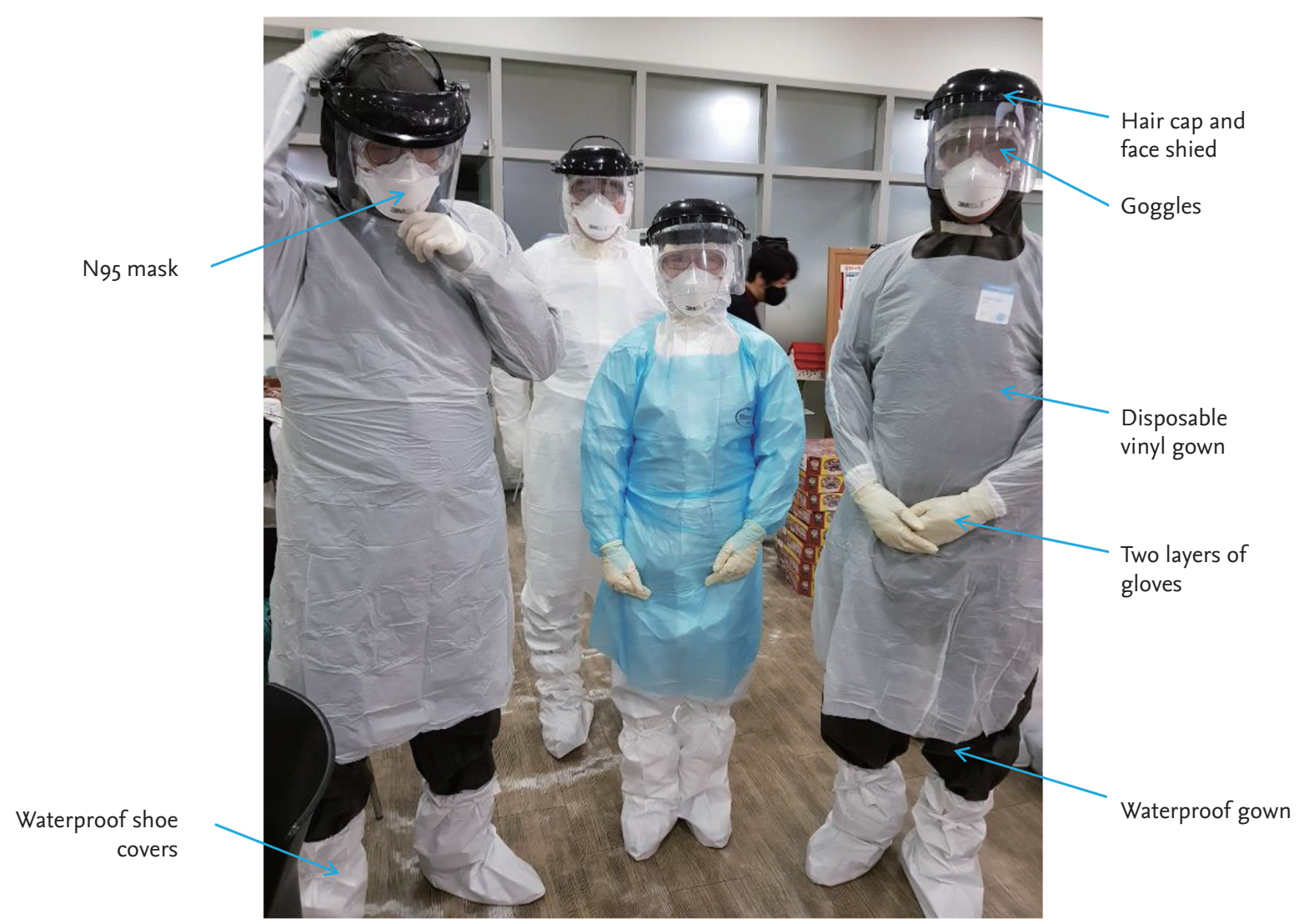

Figure 2. Personal protective equipment (PPE). During the coronavirus disease 2019 outbreak, all endoscopists and nurses in the endoscopy room wear PPE, including an N95 mask, two layers of surgical gloves, goggles, face shields, waterproof gown, and shoe covers in patients with fever, respiratory symptoms or those who had possible close contact episodes with severe acute respiratory syndrome coronavirus 2 (SARS-CoV-2)-positive patients and without SARS-CoV-2 reverse-transcription polymerase chain reaction results.

SARS-CoV-2-positive patients and without SARS-CoV-2 RT-PCR results (Fig. 2) [2-4]. As per the infection control guidelines in our center, the endoscopy room was disinfected and ventilated for an hour after completing the endoscopy.

Patients without fever, respiratory symptoms, or a history of close contact episodes with SARS-CoV-2-positive patients underwent expected endoscopy, and medical personnel in the endoscopy unit wore personal protective equipment, including dental or N95 mask, gloves, face shield, and disposable vinyl gown. If patients with fever, respiratory symptoms, or a history of a close contact episode with SARS-CoV-2-positive patients did not require emergency endoscopy, further management was determined based on the RT-PCR results. If the patient was confirmed to have positive RT-PCR results, endos- copy was postponed until the RT-PCR results became negative. However, for patients with negative RT-PCR results, endoscopy was performed per the routine process.

This study was approved by the Institutional Review Board of the Yeungnam University Hospital (YUMC 2020-06-033). Written informed consent by the patients was waived due to a retrospective nature of our study.

In conclusion, our algorithm played an important role not only in preventing viral spread to the environment but also in determining the optimal timing for performing emergency endoscopy. Owing to the use of this simple and effective algorithm during the COVID-19 outbreak, no endoscopy-related infection to other patients or medical workers was reported in our center. 


\section{Conflict of interest}

No potential conflict of interest relevant to this article was reported.

\section{Acknowledgments}

This study was supported by a 2019 Yeungnam University Research Grant.

\section{REFERENCES}

1. Wu Z, McGoogan JM. Characteristics of and important lessons from the coronavirus disease 2019 (COVID-19) outbreak in China: summary of a report of 72314 cases from the Chinese Center for Disease Control and Prevention. JAMA 2020;323:1239-1242.

2. Musa S. Hepatic and gastrointestinal involvement in coronavirus disease 2019 (COVID-19): what do we know till now? Arab J Gastroenterol 2020;21:3-8.

3. Lui RN, Wong SH, Sanchez-Luna SA, et al. Overview of guidance for endoscopy during the coronavirus disease 2019 pandemic. J Gastroenterol Hepatol 2020;35:749-759.

4. Castro Filho EC, Castro R, Fernandes FF, Pereira G, Perazzo H. Gastrointestinal endoscopy during the COVID-19 pandemic: an updated review of guidelines and statements from international and national societies. Gastrointest Endosc 2020 Apr 5 [Epub]. https://doi.org/10.1016/ j.gie.2020.03.3854.

5. Han J, Wang Y, Zhu L, et al. Preventing the spread of COVID-19 in digestive endoscopy during the resuming period: meticulous execution of screening procedures. Gastrointest Endosc 2020 Apr 5 [Epub]. https://doi. org/10.1016/j.gie.2020.03.3855. 